\title{
Preface: Rehabilitation with a Focus on Pathologies that Significantly Impact the Quality of Life
}

The current issue of this journal examines research and reviews pertinent to rehabilitation from several countries in the world, with a focus on pathologies that significantly impact the quality of life and rehabilitation, and which provides hope for relief from suffering.

Following reconstruction of injured anterior cruciate ligament after an injury, patients often wish to return to their favorite sport. However, how quickly the patient can return to the previous level of his or her sport depends on several factors. Dr. Mathias Kristiansen and his team report that problems secondary to ACL injury which include net hip internal rotator moment impulse, greater frontal plane knee range of motion, greater knee moment asymmetry in sagittal plane, and deficits in postural stability can delay and affect a complete rehabilitation. Also, patients may be classified as high risk based on their performance in the triple hop for distance. Furthermore, they report that a low quadriceps-strength limb-symmetry index can predict acute knee re-injuries.

Working in a fish-processing industry can often lead to work-related musculoskeletal disorders (WMSDs) such as tension at the neck, tendinitis, acromioclavicular syndrome, epicondylitis, and repeated stress injury, among others. Dr. Navada and his team reviewed literature on the prevalence of MSDs and their risk factors. They occur more in the upper quadrant due to high-risk movements. Workers older than 45 years, with job experience for 3 years or more combined with repetitiveness at the job, are at an increased probability of musculoskeletal disorders. Ergonomically designed work places can potentially alleviate some of the musculoskeletal issues associated with industrial fish-processing plants.

A clinical study by Yaskai and his colleagues compared the effects of positional release therapy (PRT) and lumbar stabilization exercises (LSEs) in individuals with chronic low back pain. The study was conducted on two groups: 21 participants each in the PRT and LSEs groups and 20 participants in the control group. Participants were assessed at baseline and 8 weeks following the interventions. The results suggest that both PRT and LSEs were effective in the management of individuals with chronic low back pain. The control group of patients did not show a change in their pain index. However, neither of the techniques was significantly better than the other in the management of these individuals.

Knee arthroplasty, the surgical reconstruction of the knee, is a common procedure. More than 4.7 million Americans are living with an artificial knee following knee arthroplasty. Rehabilitation after surgery is necessary to achieve maximum joint motion, pain control, and improvement in muscle strength. A review by N. Kamath and her team highlights a variety of procedures, including physical therapy, neuromuscular electrical stimulation, cold compress, balance training, exercise, and emotional support. 
Rehabilitation is crucial in restoring muscle, increasing mobility, and decreasing postsurgical pain, and it enables patients to return to many everyday activities.

The alleviation of pain is a huge challenge in the management of peripheral diabetic neuropathy. Dr. Maiyya and his team investigated the effect of sequential contraction compression therapy on pain and walking performance. Based on the findings of a before-and-after study conducted with 20 patients with diabetic neuropathy, these researchers demonstrated a significant reduction in pain measured on a visual analogue scale and improvement in walking distance.

Restoration of upper-extremity function with integration of bilateral tasks is of great importance in rehabilitating stroke survivors to achieve maximal independence in daily living activities. Dr. Kaur and her team report that both motor relearning program and bilateral arm training are effective in improving grasp, grip, pinch, and gross movement components necessary for upper extremity function.

Abdelfettah et al. report an interesting observation describing isokinetic knee muscle strength among amateur soccer and rugby players. Regardless of the anthropometric characteristics, they detected differences in isokinetic strength depending on the function occupied on the field by the athlete. In soccer players, the torque of the nondominant knee extensors of goalkeepers and defenders at high-speed in one group was statistically higher than the torque of strikers and midfielders at high-speed in the other group. Likewise, in rugby players, the torque at low-speed of the nondominant knee extensors of upper front-line players was statistically higher than the similar torque of the backline players.

Rajani Mullerpatan, $\mathrm{PhD}$

Guest Editor, Professor-Director, Mahatma Gandhi Mission School of Physiotherapy, Mahatma Gandhi Mission Institute of Health Sciences, Navi Mumbai, India

Markad V. Kamath, PhD

Editor-in-Chief 